\title{
ИНТЕРПРЕТАЦИЯ РЕЗУЛЬТАТОВ ЭЛЕКТРОПРОФИЛИРОВАНИЯ С ИСПОЛЬЗОВАНИЕМ МЕТОДА ЭМПИРИЧЕСКОЙ МОДОВОЙ ДЕКОМПОЗИЦИИ (ЕМD)
}

\author{
Л.А. ХРИСТЕНКО \\ Горный институт УрО РАН, г. Пермь
}

\begin{abstract}
Аннотация: Описаны результаты вычислительных экспериментов по разложению электрометрических данных (значений кажущегося сопротивления - КС и потенциала естественного поля - U EП) методом эмпирической модовой декомпозиции (EMD). EMD высоко адаптивный метод анализа нелинейных и нестационарных сигналов позволяет учесть все его локальные особенности, внутреннюю структуру, наличие нежелательных особенностей. Выполнено сопоставление полученных результатов с результатами, полученными ранее при использовании быстрого вейвлет-преобразования (БВП) дискретных значений кажущегося сопротивления. БМП использовалось для более отчетливого выделения скрытых закономерностей изменения амплитуды поля КС. В скользящем окне программным комплексом «КОСКАД-3D» рассчитывались статистические характеристики U ЕП и восстановленных после БВП значений КС. Рассчитанные статистики использовались в качестве вспомогательных признаков для безэталонной классификации данных электрометрических наблюдений при решении задач картирования пространственных границ участков инженерно-геологических осложнений. Используемая программа декомпозиции «RIMF» позволила выполнить разложение графиков значений КС и потенциала ЕП на IMF - функции и остатки r, из которых можно сформировать фоновые составляющие или просто очистить от помех графики наблюденных значений. Для выяснения эффективности использования EMD для мониторинговых наблюдений были выполнены расчеты для двух этапов измерений - весенних и осенних. Полученные компоненты, конечно, не отражают реальные физические процессы, но, как любые преобразования, помогают лучше понять структуру входной последовательности и упростить ее анализ, а в конечном итоге интерпретация многомерных данных по формальным признакам позволяет повысить достоверность выводов о геологической природе изучаемых явлений и процессов.
\end{abstract}

Ключевые слова: метод эмпирической модовой декомпозиции; электропрофилирование; кажущееся сопротивление; потенциал естественного поля.

Эмпирическая модовая декомпозиция (EMD) является важнейшей составляющей преобразования Гильберта-Хуанга (Huang-Hilbert Transform - HНT) [13], получившего широкое применение в различных областях науки и техники $[1,2,4,8]$, наряду с преобразованием Фурье и вейвлет-анализом. Преобразование ННТ производится в два этапа. Сначала при помощи алгоритма EMD находятся эмпирические моды или внутренние колебания (Intrinsic Mode Functions, IMF). На втором этапе при помощи преобразования Гильберта на их основе определяется мгновенный спектр исходной последовательности данных. Преобразование Гильберта-Хуанга позволяет находить мгновенный спектр нелинейных нестационарных последовательностей данных. На основе применения метода EMD можно пытаться выявить скрытые особенности взаимосвязи геофизических полей и аномалиеобразующих геологических объектов [3]. В определенных диапазонах пространственных частот эти особенности могут проявляться достаточно отчетливо, поэтому целесообразно проводить сопоставление IMF, характеризующих аномалии и их источники.

Ранее была исследована эффективность применения быстрого вейвлет-преобразования (БВП) в качестве процедуры предварительной подготовки данных электрометрических наблюдений для дальнейшего вычисления оценок их статистических характеристик и использования классификационных процедур для решения задач картирования пространственных границ участков инженерно-геологических осложнений [12, 14]. Использование процедуры БВП позволяло более отчетливо выделить скрытые закономерности изменения амплитуды поля кажущегося сопротивления (КС). 
Выполнение БВП для сглаживания графиков наблюденных значений потенциала естественного поля (U EП) не давало желаемого результата. Для графиков КС тоже не всегда подходит ортогональный вейвлет Хаара, реализованный в программе HAAR_2 [11] и используемой автором при вычислениях. То есть необходимо иметь набор вейвлет-функций, или другое - более универсальное преобразование, использование которого, как процедуры БВП, будет в конечном итоге способствовать повышению достоверности интерпретации. Общеизвестно, что интерпретация данных полевых измерений методами электропрофилирования чаще всего основана на простейших приемах оценки глубин и размеров тел или на качественном анализе. Такой способ влечет за собой неоднозначность выводов о наличии и природе явлений, вызвавших изменения наблюдаемых параметров.

В отличие от вейвлет-преобразования, в процессе эмпирической модовой декомпозиции производится разложение на некоторое число функций (IMF), которые не заданы аналитически и определяются исключительно самой анализируемой последовательностью данных. Базисные функции EMD преобразования формируются адаптивно, непосредственно из входных данных. Вейвлет-преобразование, так же как и преобразование Фурье, производит разложение в фиксированном базисе функций. Этот базис должен быть предварительно задан, то есть должна быть выбрана конкретная вейвлет-функция, используемая в процессе преобразования.

Как показали результаты вычислительных экспериментов, программа «RIMF» [7], позволяет выполнить разложение графиков значений КС и потенциала ЕП на IMF функции и остаток $\mathrm{r}$, из которых можно сформировать фоновые составляющие или просто очистить от помех графики наблюденных значений. Чтобы выяснить эффективность использования EMD для серии наблюдений, например, мониторинговых, были выполнены расчеты для двух этапов измерений. В процессе декомпозиции графиков значений КС и U ЕП, построенных по наблюдениям, выполненным в весенний период, были выделены шесть функций IMF и остаток $\mathrm{r}$ для графика КС (рис. 1a) и одна функция IMF, и остаток r для графика значений потенциала ЕП (рис. 1б). На рис. 1 все составляющие представлены в едином масштабе, что позволяет оценить вклад каждой IMF в исходный график.

a

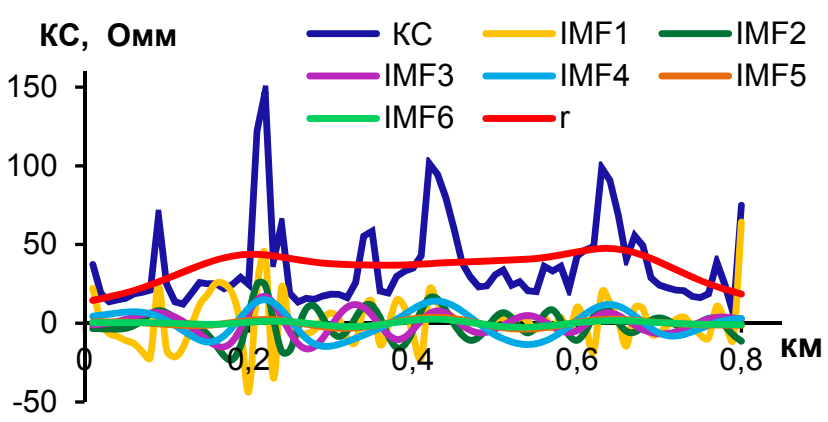

6

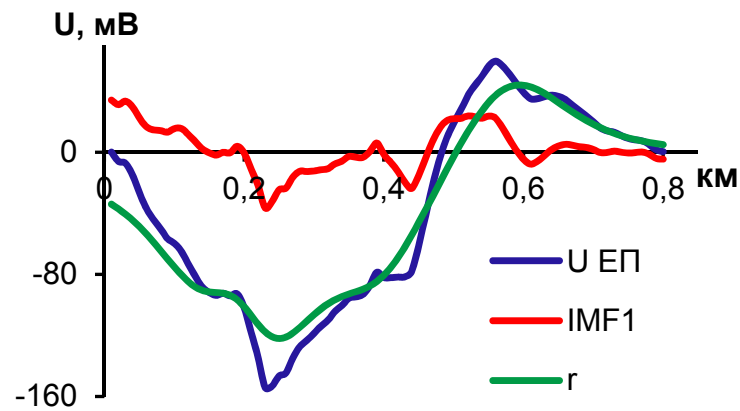

Рис. 1. Разложение графиков значений кажущегося сопротивления (а) и потенциала естественного поля (б) на функции IMF и остаток r

Декомпозиция графиков значений КС и U ЕП, построенных по наблюдениям, выполненным в осенний период, позволила выделить три функции IMF и остаток $\mathrm{r}$ для графика КС, и одну функцию IMF и остаток $\mathrm{r}$ для графика значений потенциала ЕП. Для подавления эффекта микширования мод, которое хорошо просматривается при отображении функций IMF графика КС весенних наблюдений в индивидуальном масштабе (рис. 2), использовался уже описанный в $[9,10]$ прием: вычислялись парные 
коэффициенты линейной корреляции (табл.) и суммировались функции IMF4, IMF5 и IMF6, имеющие тесную корреляционную связь. В дальнейшем сумма этих функций использовалась, как один признак - IMF4-6. Значимые коэффициенты парной линейной корреляции выделены в таблице жирным шрифтом.

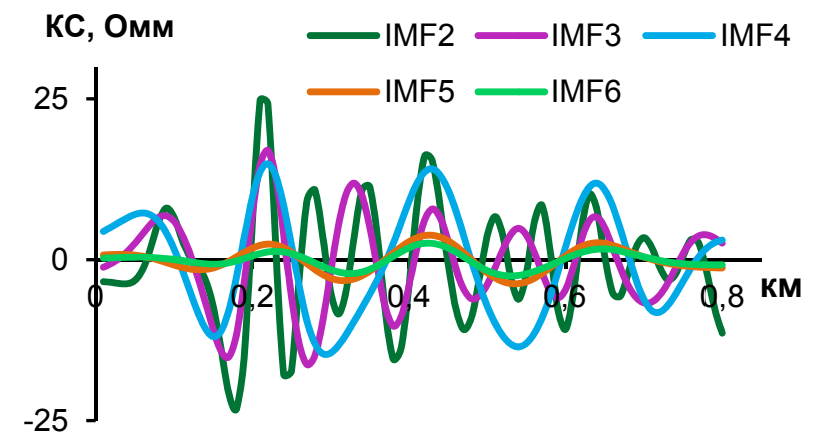

Рис. 2. Функции IMF2- IMF6 графика КC

Таблица

Парные коэффициенты корреляции IMF функций разложения графика КС

\begin{tabular}{|l|c|c|c|c|}
\hline & IMF_2 & IMF_3 & IMF_4 & IMF_5 \\
\hline IMF_2 & 1 & & & \\
\hline IMF_3 & 0.520 & 1 & & \\
\hline IMF_4 & 0.183 & 0.440 & 1 & \\
\hline IMF_5 & 0.085 & 0.109 & $\mathbf{0 . 8 9 2}$ & 1 \\
\hline IMF_6 & 0.112 & 0.080 & $\mathbf{0 . 8 5 3}$ & $\mathbf{0 . 9 7 7}$ \\
\hline
\end{tabular}

Составляющие, полученные при декомпозиции наблюденных в весенний период графиков КС и U ЕП, объединялись по две в различном сочетании:

a) функция IMF2 графика КС и остаток r графика U EП;

б) функция IMF3 графика КС и остаток r графика U EП;

в) функция IMF4-6 (сумма функций IMF4, IMF5 и IMF6) графика КС и остаток r графика U ЕП;

г) остаток r графика КС и остаток $\mathrm{r}$ графика U ЕП.

Структура полученных двухмерных совокупностей значений признаков анализировалась методом динамических сгущений (k-средних) и разбивалась на классы с помощью модуля «Коскад-профиль» программного комплекса «КОСКАД-3D» [6].

На рис. 3 приведены результаты классификации функции IMF4-6 графика КС и остатка $\mathrm{r}$ графика U ЕП (вариант в). Вероятно, из-за небольшого количества признаков, включенных

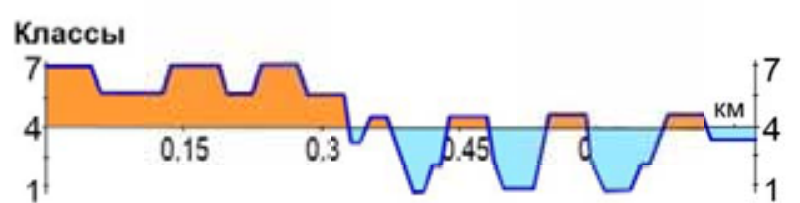

Рис.3. Результаты классификации функции IMF4-6 графика КС и остатка г графика U EП

в признаковое пространство для выполнения безэталонной классификации, сложно определить содержательный смысл интервалов, отнесенных по совокупности свойств к одному из 7 классов.

Анализ статистических характеристик с помощью аппарата теории оценок позволяет существенно увеличить объем полезной информации и более четко проследить неявно выраженные в наблюденных полях особенности геологического строения [5]. Возможно, статистические характеристики EMDфункций также содержат дополнительную полезную информацию. Для проверки этого предположения в скользящем окне по профилю были рассчитаны оценки статистических характеристик функций (среднее, дисперсия, асимметрия, эксцесс, стандарт - 5 признаков), полученных в результате EMD преобразований. Затем они объединялись в разном сочетании в многопризнаковые пространства по 10 признакам:

a) оценки статистик функции IMF2 графика КС и оценки статистик остатка $\mathrm{r}$ графика U ЕП;

б) оценки статистик функции IMF3 графика КС и оценки статистик остатка $\mathrm{r}$ графика U ЕП;

в) оценки статистик функции IMF4-6 (сумма функций IMF4, IMF5 и IMF6) графика КС и оценки статистик остатка $\mathrm{r}$ графика U EП;

г) оценки статистик остатка r графика КС и оценки статистик остатка r графика U ЕП. 
Для новых совокупностей из 10 признаков заново выполнялись процедуры безэталонной классификации.

Составляющие, полученные при декомпозиции наблюденных в осенний период графиков КС и U ЕП, тоже объединялись по две в различном сочетании и анализировались методом динамических сгущений. Для IMF-функций графиков осенних наблюдений также рассчитывались оценки статистических характеристик, объединялись в разном сочетании по 10 признаков, с которыми затем выполнялись классификационные процедуры.

Для сравнения на рис. 4 приведены некоторые результаты классификаций многопризнаковых совокупностей (по 10 признакам), выполненных после быстрого вейвлет-преобразования наблюденных значений КС (рис. 4а,б) и после преобразования EMD наблюденных значений КС и U ЕП (рис. 4в,д).

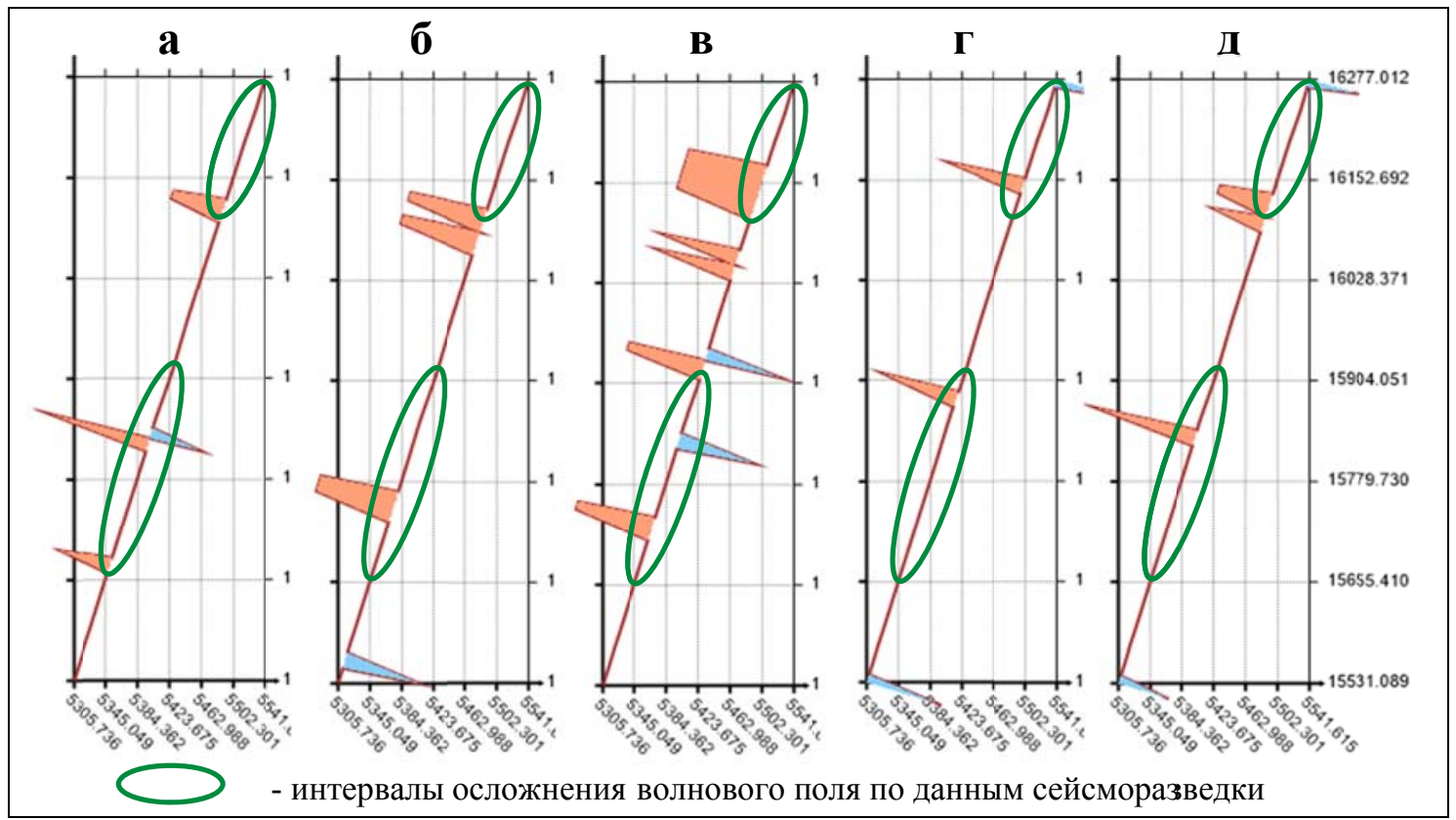

Рис. 4. Результаты классификаций по статистикам: КС (после БМПпреобразования) и $\mathrm{U}$ ЕП, полученным весной (а) и осенью (б); суммы функций IMF4, 5, 6 КС и остатка $\mathrm{r}$ U EП весенних наблюдений (в); функций IMF3 KC и IMF1 U ЕП осенних наблюдений (г); IMF3 КС и r U ЕП осенних наблюдений (д)

На рисунке (4а,в) видно, что результаты классификаций, полученные по весенним наблюдениям, заметно отличаются при использовании разных преобразований. При классификации статистик функций EMD получено большее количество интервалов, отличающихся по свойствам от фонового класса. Результаты классификаций, полученные по осенним наблюдениям, при использовании разных преобразований заметных отличий не имеют (рис. 4б,г,д). Преобразование EMD более чувствительно, чем ортогональный вейвлет Хаара, что позволило выявить скрытые в наблюденном поле особенности, вероятно, вызванные активными в весенний период фильтрационными процессами. Уже на стадии декомпозиции весенних наблюдений КС были выделены шесть функций IMF, a для осенних - три. Конечно, полученные компоненты не отражают реальные физические процессы, но, как любые преобразования, помогают лучше понять структуру входной последовательности и упростить ее анализ, а в конечном итоге интерпретация многомерных данных по формальным признакам позволяет повысить достоверность выводов о геологической природе изучаемых явлений и процессов. 
Любые преобразования требуют проверки на соответствие содержательному смыслу. Результаты классификаций на исследуемом профиле достаточно хорошо согласуются с данными сейсморазведки, по которым были выделены интервалы осложнений волнового поля в соляно-мергельной толще (СМТ). Вдоль профиля исследования СМТ прослеживается от поверхности до глубины 90 м.

Использовать результаты преобразования ЕМД при интерполяции сближенных профилей (вариант 2D) для выделения границ возможных инженерно-геологических осложнений по единым критериям очень сложно из-за различий частотно-амплитудных характеристик полученных IMF-функций наблюденных параметров. Для проверки достоверности результатов представленную последовательность преобразований следует еще неоднократно опробовать и сопоставить с результатами интерпретации других методов исследования.

\section{Работа выполнена при поддержке РФФИ (проект № 19-05-00654 А).}

\section{БИБЛИОГРАФИЧЕСКИЙ СПИСОК}

1. Велигура С.А. Анализ и моделирование электропотребления с помощью метода эмпирической модовой декомпозиции // Современные энергетические системы и комплексы и управление ими: Материалы 14-ой Междунар. науч.-практич. конф. - Новочеркасск, 2018. - С. 92-94.

2. Дюк В.А., Комашинский В.И., Малыгин И.Г., Сенкевич Ю.И., Аванесов М.Ю. Исследование эмпирической модовой декомпозиции в задаче анализа сигналов акустической эмиссии // Информация и космос. - 2018. - № 4. - С. 50-55.

3. Калинин Д.Ф., Яновская Ю.А., Долгаль А.С. Результаты профильной комплексной интерпретации геопотенциальных полей методом эмпирической модовой декомпозиции (EMD) с целью оценки перспектив нефтегазоносности // Геофизика. - 2019. - № 1. - С. 2-12.

4. Омпоков В.Д., Бороноев В.В. Исследование частотно-временных характеристик пульсовых сигналов с помощью преобразования Гильберта-Хуанга // Журнал радиоэлектроники. - 2017. - № 5. - С. 10.

5. Петров А.В. Теоретические основы обработки геофизических данных: Учеб. пособие. - М., 2004. - 37c.

6. Петров А.В., Юдин Д.Б., Хоу Сюели Обработка и интерпретация геофизических данных методами вероятностно-статистического подхода с использованием компьютерной технологии «КОСКАД 3D» // Вестник КРАУНЦ. Науки о Земле. - 2010. - № 2, Вып.16. - С. 126-132.

7. Христенко Л.А. Метод эмпирической модовой декомпозиции при выделении информативных компонент результатов электропрофилирования // Стратегия и процессы освоения георесурсов: сб. науч. тр. Вып. 16 / ГИ УрО РАН. - Пермь, 2018. - С. 233-237. DOI: 10.7242/gdsp/2018.16.64

8. Ястребов И.П. О свойствах и применениях преобразования Гильберта-Хуанга // Проектирование и технология электронных средств. - 2016. - № 1. - С. 26-33.

9. Долгаль А.С. Учет влияния рельефа земной поверхности при отработке результатов измерений магнитного поля // Вопросы теории и практики геологической интерпретации геофизических полей: материалы 45-й сес. Междунар. семинара им. Д.Г. Успенского / Казан.фед. ун-т. - Казань, 2018. - С. 134-136.

10. Dolgal A.S., Khristenko L.A. Application of empirical mode decomposition method in processing geophysical data // Bulletin of the Tomsk Polytechnic University, Geo Assets Engineering. - 2017. - № 1. - P. 100-108.

11. Долгаль А.С., Симанов А.А. Применение кратномасштабного вейвлет-анализа при аналитических аппроксимациях геопотенциальных полей // Докл. Акад. наук. - 2008. - Т. 418, № 2. - С. 256-261.

12. Hristenko L., Stepanov Yu., Kichigin A., Parshakov E., Tainitsky A. Interpretation of electroprospecting monitoring observations with use of probabilistic-statistical characteristics // Engineering and mining Geophysics 2018: 14th Conference \& Exhibition, 23-27 april 2018. - Almaty, 2018. - code 137600. DOI: 10.3997/2214-4609.201800511.

13. Huang N.E., Shen Z., Long S.R., Wu M.C., Shih H.H., Zheng Q., Yen N.-C., Tung C.C., Liu H. H. The empirical mode decomposition and the Hilbert spectrum for nonlinear and non-stationary time series analysis // Proceedings of the royal society A-Mathematical physical and engineering sciences. - 1998. - V. 454, № 1971. - P. 903-995. DOI: 10.1098/rspa.1998.0193.

14. Khristenko L.A., Stepanov Ju.I., Kichigin A.V., Parshakov E.I., Tainickiy A.A., Shiryaev K.N. Using of Probabilistic-Statistical Characteristics in the Interpretation of Electrical Survey Monitoring Observations // Practical and Theoretical Aspects of Geological Interpretation of Gravitational, Magnetic and Electric Fields: Proceedings of the 45th Uspensky International Geophysical Seminar, Kazan, Russia / ed. D. Nurgaliev, N. Khairullina; Kazan Federal University. - Springer, Cham, 2019. - P. 313-320. - (Book series: Springer Proceedings in Earth and Environmental Sciences). 